\section{Neues Mehrkomponenten-Spritzgerät für Brandschutzbeschichtungen}

$\mathrm{B}$ randschutzanstriche aus Epoxyd schützen Stahl vor kritischen Temperaturen bei Bränden mit kohlenwasserstoff- beziehungsweise zellulose-

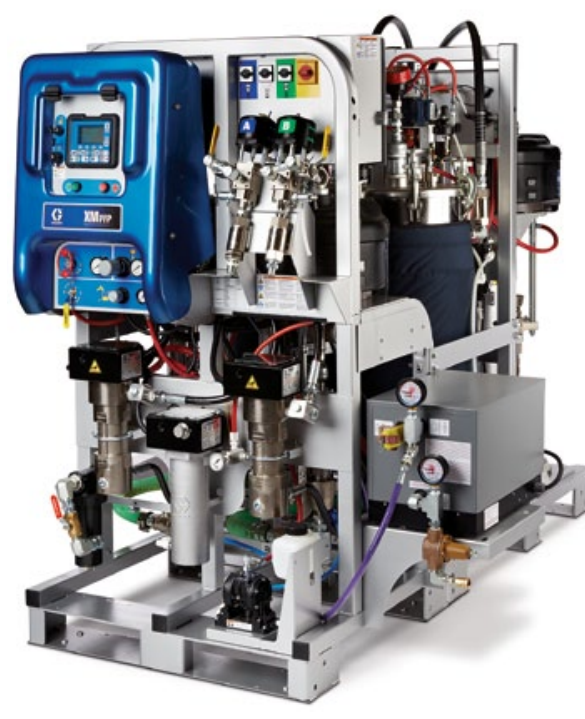

haltigen Stoffen. Kritische Anwendungen erfordern Auftragstechnologien für diese Anstriche, die präzise $\mathrm{Mi}$ schungsverhältnisse liefern. Das Mehrkomponenten-Spritzgerät XM PFP von Graco ist auf maximale Produktivität, höchste Leistung und ein präzises Mischungsverhältnis ausgelegt. Das Mischungsverhältnis ist einstellbar, somit entfallen teure Umbauarbeiten zwischen Aufträgen mit unterschiedlichen Beschichtungen. Die Datenprotokollierung per USB ermöglicht eine optimale Qualitätskontrolle. Zudem sorgen neue Temperatursteuerungen und Heizsysteme für eine verbesserte Spritzleistung und höhere Produktivität. Anwender sind somit in der Lage, die Beschichtungsmaterialien bei möglichst niedrigen Drücken zu zerstäuben. Dies bedeutet weniger Abfall und Overspray.

Tel. +3289 770 700, www.graco.com

\title{
Erweiterte Produktlinie mit Automatikpistolen
}

$M$ it Einführung der neuen Serie TOF-B hat die Anest lwata $\mathrm{GmbH}$ ihre Automatik-Lackierpistolen-Produktlinie auf insgesamt 6 Typen und 26 Modelle erweitert. Verwendet werden die Automatik-Lackierpistolen beispielsweise feststehend an Balken für die Beschichtung durchlaufender Werkstücke, in der Inline-Beschichtung hinter Extrudern für Gummioder Kunststoffprofile sowie in der Randbeschichtung bei Holz- oder Baustoffplatten. Die Anwendungsbereiche der TOF-Serie reichen von der Kunststoffherstellung über die Baustoffindustrie bis hin zur Holz- und Papierindustrie. Verfügbar ist die neue TOF-B-Serie als einfacher Typ mit einer gemeinsamen Luftquelle für die Steuer- und Zerstäuberluft sowie als Standard-Typ mit zwei separaten Luftquellen für die Steuer- und Zerstäuberluft. Der einfache Typ ist aufgrund der reduzierten Anschlüsse problemlos an Maschinen zu montieren. Beim Standard-Typ hingegen ist durch die se- parate Luftquelle eine Anpassung der Zerstäuberluft möglich, um ein optimales Ergebnis zu erreichen. Tel. 0341241443 0, www.anest-iwata.de

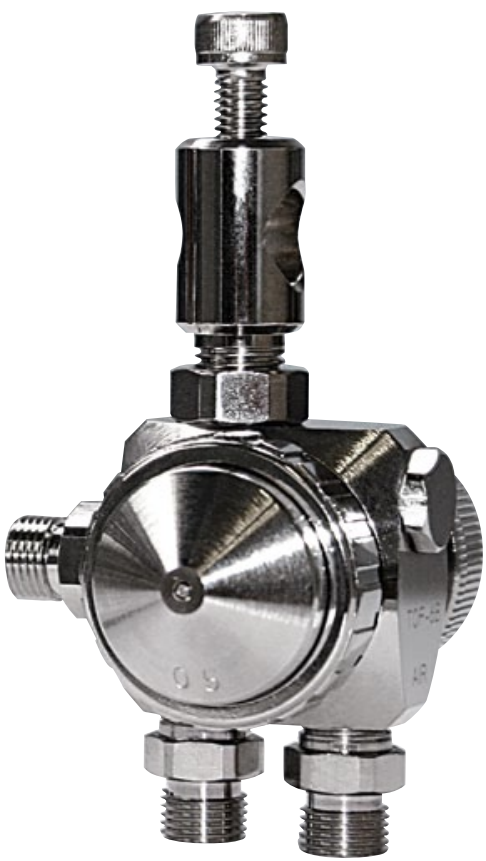

Partikel- und

FlüssigkeitstropfenAbscheidung mit einem Filter

Eür die Abscheidung von Wasser- tropfen wie Nebel und Aerosole sowie von schwebenden Partikeln aus der Luft bietet Wiltec neue eigensteife Taschenfilter an, die so

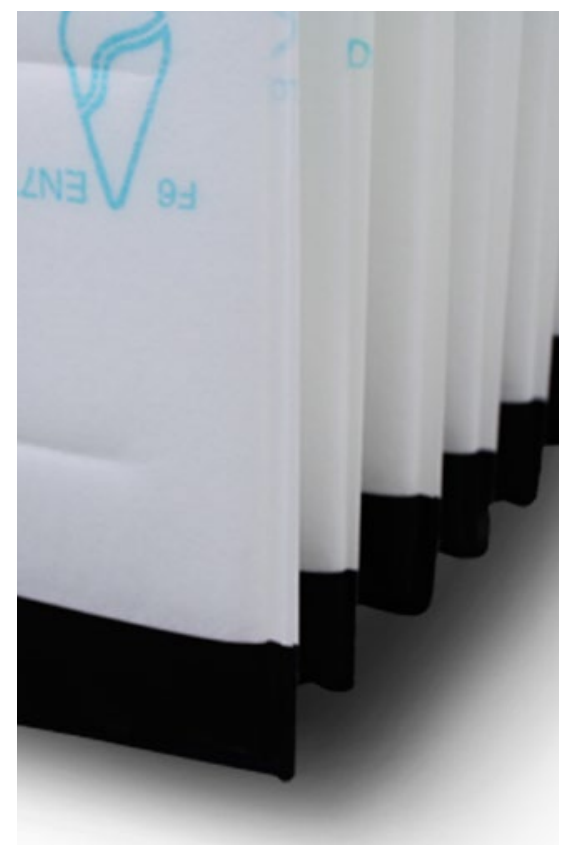

ausgerüstet sind, dass Wasser abgewiesen wird und abgeschiedener Staub haften bleibt. Die Drop-Safe-Taschenfilter sind besonders geeignet für extreme Umweltbedingungen wie zum Beispiel sehr hohe Feuchtigkeit sowie hohe Wasseraerosol-Konzentrationen und Luftgeschwindigkeiten. Die Filter sind in Filterklassen G4, F5 und F6 gemäß EN 779 erhältlich und ermöglichen durch die progressive FaserdichteStruktur eine sehr gute Staubspeicherung. Die eigensteifen, leck-freien Taschen behalten auch in turbulenter Luft und durchnässt ihre Form. Die Taschen sind in einen schlagfesten PU-Rahmen eingespritzt, wasserdicht und berst-sicher bis $\geq 6000 \mathrm{~Pa}$. Die Filter können durch Verbrennung entsorgt werden.

Vertrieb durch Wiltec,

Tel. +31 4132444 44, www.wiltec.nl 